\title{
Hilbert's Sixteenth Problem for polynomial Liénard equations
}

\author{
Magdalena Caubergh
}

\begin{abstract}
This article reports on the survey talk 'Hilbert's Sixteenth Problem for Liénard equations,' given by the author at the Oberwolfach MiniWorkshop 'Algebraic and Analytic Techniques for Polynomial Vector Fields.' It is written in a way that it is accessible to a public with heterogeneous mathematical background as the one at the Mini-Workshop. The article reviews recent developments and techniques used in the study of Hilbert's 16th problem where the main focus is put on the subclass of polynomial vector fields derived from the Liénard equations.
\end{abstract}

Mathematics Subject Classification (2000). Primary 34C07; Secondary 37G15, 34C20 .

Keywords. Planar vector fields, periodic orbits, compactification, large amplitude limit cycles, Liénard equations.

\section{Introduction}

Polynomial Liénard equations are planar differential equations associated to the second order scalar differential equations

$$
x^{\prime \prime}+f(x) x^{\prime}+g(x)=0,
$$

where the functions $f$ and $g$ are polynomials of degree $n$ and $m$ respectively. They occur as models or at least as simplifications of models in many domains of science.

Besides singularities, which are well-understood for (1.1), isolated periodic orbits or so-called limit cycles represent the asymptotic state of the other solutions of (1.1), see Figure 1, which are subject of Hilbert's 16th Problem and Smale's 13th Problem. A formulation of these problems and their recent developments are recalled in section 2. There were several attempts to solve Hilbert's 16th Problem and so far all of them failed. However the problem yet has a source of inspiration for significant progress in the geometric theory of planar vector fields, as well as bifurcation theory, normal forms, foliations and some topics in algebraic geometry. 
This survey aims at collecting important developments and techniques in the study of Hilbert's 16th Problem where the main focus is put on the subclass of polynomial vector fields derived from the Liénard equations (1.1) :

$$
\dot{x}=y, \dot{y}=-g(x)-f(x) y,
$$

or written in the coordinates $(X, Y)=(x, y+F(x))$ of the Liénard plane:

$$
\dot{X}=Y-F(X), \dot{Y}=-g(X) \text { where } F(X)=\int_{0}^{X} f(u) d u \text {. }
$$

\section{Hilbert's Sixteenth Problem}

\subsection{Polynomial vector fields}

Hilbert's 16th Problem essentially asks for a uniform upper bound $\mathcal{H}(n)$ for the maximum number of limit cycles of a planar polynomial vector field

$$
X_{(a, b)}^{n} \leftrightarrow\left\{x^{\prime}=\sum_{j=0}^{n} \sum_{i=0}^{j} a_{i j} x^{i} y^{j-i} \text { and } y^{\prime}=\sum_{j=0}^{n} \sum_{i=0}^{j} b_{i j} x^{i} y^{j-i}\right\} \text { where } a_{i j}, b_{i j} \in \mathbb{R}
$$

uniformly in terms of the degree $n$. This problem is more than 100 years old and its investigation has produced many papers contributing to the wide development of the theory of Dynamical Systems. It is not known whether a uniform upper bound only depending on the degree of the vector field might exist, even not when the degree is two. This part of Hilbert's 16th Problem is generally called the uniform finiteness problem.

Even Dulac's theorem to prove that for individual vector fields the number of limit cycles is finite was far from trivial (see e.g. [18]); this problem is also referred to as the individual finiteness problem. In 1923 Dulac presented a proof for this theorem. Later it was found that the proof contained a gap. This lack was first solved for quadratic systems by Bamon in 1985 and for arbitrary degree by Ecalle and Ilyashenko independently in the 1990s.

Solution programmes for Hilbert's 16th Problem mostly consist in its reduction to several subproblems, based on either considering local cyclicity problems [26] or restricting the class of vector fields to a particular simpler class, see e.g. [18] for an overview.

Using different techniques (Abelian integrals, simultaneous bifurcations from centers,... ), there are some lower estimates known for the Hilbert's numbers, for instance: $\mathcal{H}(2) \geq 4[9,29,33], \mathcal{H}(3) \geq 13[23], \mathcal{H}(4) \geq 22[2], \mathcal{H}(5) \geq 28[30]$, $\mathcal{H}(6) \geq 35[31], \mathcal{H}(7) \geq 50[25], \mathcal{H}(n) \geq k n^{2} \ln n[7]$, and

$$
\mathcal{H}(n) \geq 4(n+1)^{2}(1.442695 \ln (n+1)-1 / 6)+n-2 / 3[20] .
$$

Finally note that Hilbert's 16th problem was also considered for vector fields on phase spaces of arbitrary dimension $\geq 3$ by Bobienski and Zoladek; in [1] they illustrate that then the uniform finiteness problem has a negative answer. 


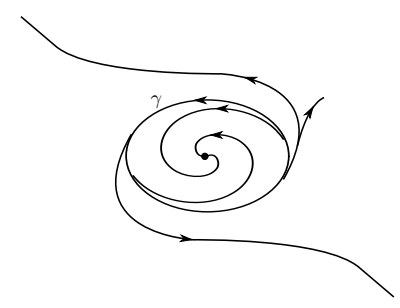

Figure 1. Repelling limit cycle $\gamma$.

\subsection{Liénard equations}

In the following we denote by $\mathcal{H}_{L}(m, n)$ the maximum number of limit cycles of (1.1), (1.2) or (1.3). Part of the 13th Problem that Smale put forward on his list of challenging problems for the 21st century deals with Hilbert's 16th Problem restricted to the classical Liénard equations, i.e. the case $g(x)=x$ in $(1.1),(1.2)$ or (1.3), see [28]. Moreover Smale suggests that the maximal number of limit cycles $\mathcal{H}_{L}(1, n)$ for classical Liénard equations grows at most by an algebraic law of type $n^{d}$ where $d$ is a universal constant.

The problem for classical Liénard equations when the degree of $f$ is equal to 2 or 3 is solved; the result in [24] shows that $\mathcal{H}_{L}(1,2)=1$ (i.e. the so-called Van der Pol Equation has at most one limit cycle, see Figure 1) and very recently Li and Llibre proved in their preprint [22] that $\mathcal{H}_{L}(3,1)=1$. Besides there is the so-called Lins, de Melo and Pugh Conjecture, stating that the maximal number of limit cycles is equal to $l$ if $g(x)=x$ and the degree of $f$ is $2 l$ or $2 l+1$.

Of course there is the counter-example to that conjecture, for limit cycles in singular perturbations, due to Dumortier, Panazzolo and Roussarie $\left(\mathcal{H}_{L}(1,6) \geq 4\right.$ see [15] and a recent generalization $\mathcal{H}_{L}(1, n) \geq\left[\frac{n}{2}\right]+2, \forall n \geq 5$ in [10]), but it does not contradict the possibility for the growth of the number of limit cycles to be linear. In [15] classical Liénard equations are presented with degree of $f$ equal to $2 l$ and having at least $l+1$ limit cycles; hence one limit cycle more than conjectured by Lins, de Melo and Pugh.

In fact, in [24], they prove that, under these assumptions, there are at most $l$ small amplitude limit cycles. Lloyd and Lynch considered the similar problem for generalized Liénard equations [21]. In most cases, they prove an upper bound for the number of small amplitude limit cycles, that can bifurcate out of a single non-degenerate singularity.

Later Coppel proved in [8] that $\mathcal{H}_{L}(2,1)=1$. In $[12,17]$ it is shown that $\mathcal{H}_{L}(3,1)=1$ and in [13] it is shown that $\mathcal{H}_{L}(2,2)=1$. Up to now, as far as we know, only these five cases have been completely investigated. From Dumortier and Li we know that $\mathcal{H}_{L}(2,3) \geq 5$ [14]; furthermore from Yang, Han and Romanovski we know that $\mathcal{H}_{L}(m, 3) \geq\left[\frac{3 m+14}{4}\right]$ for $3 \leq m \leq 8$, see [32]. 
Recently progress has been made towards proving the finiteness part of Hilbert's 16th Problem for classical Liénard equations. By the results from [4, 27] the study of the finiteness part of Smale's 13th Problem is reduced to singular perturbation problems; more precisely the following theorem is proven.

Theorem 2.1. The global number of limit cycles of (1.1) with $g(x)=x$ is uniformly bounded if $f$ is restricted to some compact set of polynomials of degree exactly $n$ (see [27] for $n$ even and [4] for $n$ odd).

In section 4 we provide with some insight in the techniques underlying Theorem 2.1. In (2.1) we have summarized the currently known results for the Hilbert numbers for Liénard equations (1.2) that we mentioned here above.

\begin{tabular}{r|r|l|r|r|r|r|l}
$\mathcal{H}_{L}(m, n)$ & 1 & 2 & 3 & 4 & 5 & 6 & $n \rightarrow$ \\
\hline 1 & 0 & 1 & 1 & & $\geq 4$ & $\geq 5$ & {$[4,27],[10]$} \\
\hline 2 & 1 & 1 & $\geq 5$ & & & & \\
\hline 3 & 1 & & & & & & \\
\hline$m \downarrow$ & & & {$[32]$} & & & &
\end{tabular}

\section{Local and global finiteness problems}

Hilbert's 16th Problem is a global finiteness problem in the sense that one aims at bounding the number of limit cycles of $X_{(a, b)}^{n}$ in the plane $\mathbb{R}^{2}$ for all possible values of the parameter $(a, b)$. In this section we briefly explain how this global problem can be 'localized'. For this purpose we first introduce the notions of limit periodic set and cyclicity. Limit periodic sets are subsets consisting of singularities and regular orbits, that can produce limit cycles by perturbation, and the cyclicity is the maximal number of limit cycles that they can generate in a perturbation. More precisely these notions are defined as:

Definition 3.1. Let $P \subset \mathbb{R}^{p}$ be the parameter space with $\lambda^{0} \in P$ and let $\left(X_{\lambda}\right)_{\lambda \in P}$ be a family of vector fields on a regular 2-dimensional surface $S$. Then,

1. a compact set $\Gamma \subset S$ is a limit periodic set of $\left(X_{\lambda}\right)_{\lambda}$ for $\lambda \rightarrow \lambda^{0}$ if and only if there exists a sequence $\left(\lambda_{n}\right)_{n \in \mathbb{N}}$ in $P$ with $\lambda_{n} \rightarrow \lambda^{0}$ for $n \rightarrow \infty$ and such that for all $n \in \mathbb{N}$, there exists a limit cycle $\gamma_{n}$ of $X_{\lambda_{n}}$ with $\gamma_{n} \rightarrow \Gamma$ for $n \rightarrow \infty$.

2. Let $\Gamma$ be a limit periodic set of $\left(X_{\lambda}\right)_{\lambda}$ for $\lambda \rightarrow \lambda^{0}$. Then we say that $\Gamma$ has finite cyclicity in the unfolding $\left(X_{\lambda}\right)_{\lambda}$ for $\lambda \rightarrow \lambda^{0}$ if there exist $N \in \mathbb{N}$ and constants $\varepsilon, \delta>0$ such that for every parameter value $\lambda$ with $\left\|\lambda-\lambda^{0}\right\|<\delta$ the vector field $X_{\lambda}$ has at most $N$ limit cycles $\gamma$ with $d_{H}(\gamma, \Gamma)<\varepsilon$. If $\Gamma$ has finite cyclicity in $\left(X_{\lambda}\right)_{\lambda}$ for $\lambda \rightarrow \lambda^{0}$, then the cyclicity of $\Gamma$ inside the unfolding $\left(X_{\lambda}\right)_{\lambda}$ for $\lambda \rightarrow \lambda^{0}$ is defined as the positive integer

$$
\operatorname{Cycl}\left(X_{\lambda},\left(\Gamma, \lambda^{0}\right)\right)=\lim _{\lambda \rightarrow \lambda^{0}} \sup _{\gamma \rightarrow \Gamma}\left\{\text { number of limit cycles } \gamma \text { of } X_{\lambda}\right\} \text {. }
$$

The distance $d_{H}$ is the Hausdorff distance on the metric space of compact sets, and the limits $\gamma_{n} \rightarrow \Gamma$ and $\gamma \rightarrow \Gamma$ are considered in this metric space. 
A major question is the so-called local finiteness problem or local cyclicity problem that exists in finding sufficient conditions under which the cyclicity is finite, and in this case, to find an explicit estimate for the cyclicity.

Limit periodic sets are the so-called organizing centers for limit cycles. Therefore to detect limit cycles one looks first for all limit periodic sets, for which there exists in case there are only isolated singularities a structure thm analogous to the Poincaré-Bendixson Theorem for $\alpha$ - and $\omega$-limit sets (see [26]). This theorem classifies the possible types of limit periodic sets $\Gamma$ of $\left(X_{\lambda}\right)_{\lambda}$ for $\lambda \rightarrow \lambda^{0}$ as a singular point, a periodic orbit or a graphic of $X_{\lambda^{0}}$. Recall that a graphic $\Gamma$ of a vector field $X_{\lambda^{0}}$ is the union of (not necessarily distinct) singular points $p_{0}, \ldots, p_{m}$ with $p_{m}=p_{0}$, and regular orbits $\gamma_{1}, \ldots, \gamma_{m-1}$ of $X_{\lambda^{0}}$, connecting these singular points, in the sense that $p_{i}=\alpha\left(\gamma_{i}\right)$ and $p_{i+1}=\omega\left(\gamma_{i}\right), 1 \leq i \leq m-1$. In section 4.3 we encounter the so-called '2-saddle cycle' which is a simple example of a graphic, see Figure $3\left(\Gamma=\Gamma_{1} \cup \Gamma_{2} \cup\left\{\mathbf{s}_{+}, \mathbf{s}_{-}\right\}\right)$.

By compactification of the parameter space and the phase plane of $X_{(a, b)}^{n}$, the family of polynomial vector fields extends to a compact analytic family, such that Hilbert's global finiteness problem is reduced to several local cyclicity problems (see Theorem 3.2 below and [27]).

\subsection{Localization method}

Compactifying phase plane as well as parameter space one can apply the so-called localization method of Roussarie $([27])$ which is based on the following theorem:

Theorem 3.2. Let $K$ be a compact set in parameter space and let $S$ be a compact 2-dimensional manifold, let $X_{\lambda}$ be an analytic vector field on $S$ for each $\lambda \in K$. Then there exists a uniform upper bound for the number of limit cycles of $X_{\lambda}$ in $S$ for $\lambda \in K$ if and only if for any $\lambda^{0} \in K$ any limit periodic set $\Gamma$ of $X_{\lambda^{0}}$ has finite cyclicity inside the family $X_{\lambda}$ for $\lambda \rightarrow \lambda^{0}$, i.e.

$$
\operatorname{Cycl}\left(X_{\lambda},\left(\Gamma, \lambda^{0}\right)\right)<\infty .
$$

In section 4 , to prove Theorem 2.1 , we apply the localization method of Roussarie reducing the global Smale's problem to several local cyclicity problems: small, medium and large amplitude limit cycles and cyclicity problems for slowfast systems. This localization method requires an appropriate compactification of the phase plane, as well as the chosen space of Liénard equations itself (see section 4.5).

\subsection{Classical tools to study local cyclicity problems}

Traditionally the study of limit cycles of planar vector fields $\left(X_{\lambda}\right)_{\lambda}$ near a limit periodic set $\Gamma$ for $\lambda \rightarrow \lambda^{0}$, as the ones we consider, is replaced by the study of isolated fixed points $s$ of associated 1-dimensional Poincaré-maps $\left(P_{\lambda}\right)_{\lambda}$,

$$
P_{\lambda}(s)=s \text { for } s \text { near } s_{0},
$$

where $s_{0}$ corresponds to $\Gamma$. Or equivalently, by the study of isolated zeroes $s$ of so-called displacement maps $\delta_{\lambda}=P_{\lambda}-I d$ or difference maps $\Delta_{\lambda}$ (e.g., see section 
4.3 and Figure 3 ). In such a way configurations of isolated zeroes of $\delta_{\lambda}$ (resp. $\Delta_{\lambda}$ ) correspond to configurations of limit cycles of $X_{\lambda}$. In particular the cyclicity in (3.1) can be expressed in terms of zeroes of $\Delta_{\lambda}$ (or $\left.\delta_{\lambda}\right)$ :

$$
\operatorname{Cycl}\left(X_{\lambda},\left(\Gamma, \lambda^{0}\right)\right)=\lim _{\lambda \rightarrow \lambda^{0}} \sup _{s \rightarrow s^{0}}\left\{\text { number of isolated zeroes } s \text { of } \Delta_{\lambda}\right\} .
$$

To control zeroes of these maps near $s^{0}$ one can rely on classical theorems as the Implicit Function theorem, Rolle's Theorem, the Preparation Theorem, as long as the map $\Delta_{\lambda^{0}}$ is of finite order at $s^{0}$. In the other case some degeneracies first have to be removed. To that end, when $\Gamma$ is approached by a period annulus, one can analyze the division of the map in terms of Melnikov functions or the Bautin ideal (see [26]). The Bautin ideal also serves to characterize the parameter values for which the corresponding vector field is of center type. Moreover an upper bound of the cyclicity can be expressed in terms of this ideal. In [3] it is proven that this Bautin ideal corresponds to the ideal generated by Lyapunov quantities, which determine the order and stability of a weak focus; in [6] Lyapunov quantities are determined for classical and generalized Liénard equations using Cherkas transformation, and a local division of the displacement map is given in terms of the Lyapunov quantities. Furthermore in [6] a detailed study of the bifurcation phenomenon of small amplitude limit cycles is provided and generic Hopf-Takens bifurcations (degenerate or not) are precisely described.

\section{Classical Liénard equations of degree $n$}

In the Liénard plane the classical Liénard equation (1.3) is written as the polynomial vector field $L_{a}^{n}$ with

$$
L_{a}^{n} \leftrightarrow \dot{x}=y-F_{a}^{n}(x), \dot{y}=-x \text { where } F_{a}^{n}(x)=\sum_{i=1}^{n-1} a_{i} x^{i}+x^{n} .
$$

Besides the compactification process, due to the fact that the 'central system' is too degenerate to permit a study of its unfolding without a blow up, the method includes a desingularization. In this way the boundary of the space of Liénard equations is made by Hamiltonian and singular perturbation problems. These boundary problems both exhibit different phenomena. In this survey we only include the Hamiltonian perturbation problems that can completely be solved. The study of the cyclicity problem for classical Liénard equations of odd degree (i.e. $n$ is odd) that do not belong to the boundary is easy and well-known among specialists. In this case limit cycles stay at a uniform distance from infinity (see [27]). For classical Liénard equations of even degree (i.e. $n=2 l$ is even) this is no longer true and then the main problem consists in studying limit cycles that come close to infinity. These limit cycles are so-called large amplitude limit cycles of which it is shown in [4] that there are at most $l+1$ (see Definition 4.1). 


\subsection{Bounded Liénard equations}

By 'bounded Liénard equations' of degree $n$ we refer to a family of Liénard equations $\left(L_{a}^{n}\right)_{a}$, where $a$ belongs to a compact set $K$ in $\mathbb{R}^{n-1}$.

Applying the Lyapunov-Poincaré compactification, that is a quasi-homogenous version of the Poincaré compactification, the Liénard system $L_{a}^{n}$ extends to an analytic vector field on the sphere $\mathbb{S}^{2}$ (see [11]). In this compactification the nonelementary singularities at infinity are spread out over the equator of $\mathbb{S}^{2}$ being all of elementary type.

\subsection{Local cyclicity problems for bounded Liénard equations}

Since the origin is the only singularity of $L_{a}^{n}$ in $\mathbb{R}^{2}$, that is a weak focus or center, limit periodic sets that lie entirely in the finite plane correspond to either the singularity in the origin or a regular periodic orbit. Limit cycles bifurcating from these limit periodic sets are called respectively small amplitude limit cycles and medium amplitude limit cycles. These cyclicity problems are well understood and result in finite cyclicity, because these limit cycle bifurcations can be studied by isolated zeroes of analytic maps (displacement maps), see e.g. [6].

Since there are no other limit periodic sets in the finite plane the only bifurcation phenomenon of limit cycles that remains to be studied is the one of large amplitude limit cycles, that is defined here below. Let $B_{R}(0)$ denote the open ball in $\mathbb{R}^{2}$ centered at $(0,0)$ and with radius $R>0$ :

$$
B_{R}(0)=\left\{(x, y) \in \mathbb{R}^{2}: x^{2}+y^{2}<R\right\} .
$$

Definition 4.1. We say that a family $\left(X_{\lambda}\right)_{\lambda}$ of vector fields on the plane $\mathbb{R}^{2}$ has exactly $N$ large amplitude limit cycles for $\lambda \rightarrow \lambda^{0}$ if and only if

1. there exist $R>0$, a neighbourhood $W$ of $\lambda^{0}$ such that for all $\lambda \in W$ the vector field $X_{\lambda}$ has at most $N$ limit cycles having a non-empty intersection with $\mathbf{R}^{2} \backslash B_{R}(0)$;

2. for all $R>0$ and for every neighbourhood $W$ of $\lambda^{0}$, there exists $\lambda \in W$ such that $X_{\lambda^{0}}$ has $N$ limit cycles having a non-empty intersection with $\mathbf{R}^{2} \backslash B_{R}(0)$.

In sections 4.3 and 4.4 the proofs will be sketched of the facts that no large amplitude limit cycles appear for the family of Liénard equations of odd degree, while there appear at most $l+1$ for Liénard equations of even degree $n=2 l$.

\subsection{No large amplitude limit cycles in case $n$ is odd}

For $n$ is odd one can construct a uniform domain of attraction by considering the Lyapunov function $V(x, y)=\left(x^{2}+y^{2}\right) / 2$. Since the time derivative of $V$ is given by

$$
\dot{V}(x, y)=x \dot{x}+y \dot{y}=-x F_{a}^{n}(x)=-x^{n+1}(1+o(1)), x \rightarrow+\infty .
$$

Therefore given an arbitrary compact set $K \subset \mathbf{R}^{n-1}$ there exists $R_{K}>0$ such that for all $a \in K$ all orbits of $L_{a}^{n}$ enter $\bar{B}_{R_{K}}(0)$ after some finite time, and stay there for all later times. As a consequence there are no large amplitude limit cycles. 


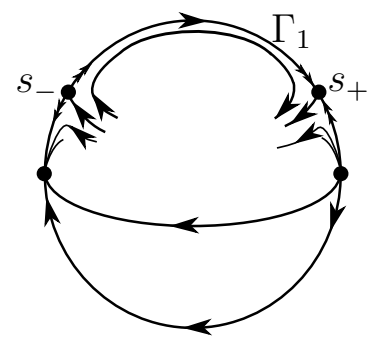

(a)

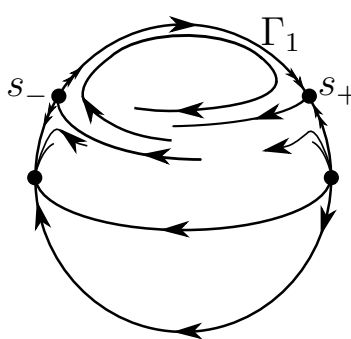

(b)

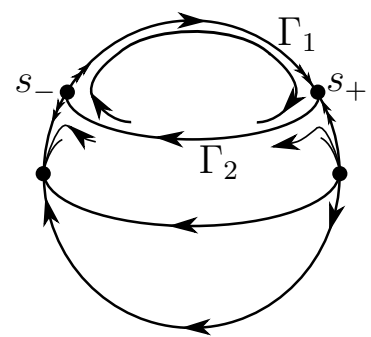

(c)

Figure 2. Classical Liénard equation $L_{a}^{2 l}$ extended to the Poincaré-Lyapunov disc of type $(1,2 l)$. (a) Topological behavior at $\infty$. (b) No connection between $\mathbf{s}_{+}$and $\mathbf{s}_{-}$in the finite plane. (c) Connection $\Gamma_{2}$ between $\mathbf{s}_{+}$and $\mathbf{s}_{-}$in the finite plane, thus giving rise to the unbounded 2 -saddle cycle $\Gamma=\Gamma_{1} \cup \Gamma_{2} \cup\left\{\mathbf{s}_{+}, \mathbf{s}_{-}\right\}$.

\subsection{At most $l+1$ large amplitude limit cycles in case $n$ is even}

For $n=2 l$ even, the infinity is no longer repelling and we here sketch briefly how the analysis of large amplitude limit cycles proceeds.

1. Compactification of phase space: take local coordinates $(\bar{x}, \bar{y})$ near infinity given by $x=\bar{x} / s, y=\bar{y} / s^{2 l}$ with $s>0$ and $\bar{x}^{2}+\bar{y}^{2}=1$. After a time-rescaling $\left(\bar{t}=s^{2 l-1} t\right)$ the vector field $L_{a}^{n}$ extends analytically to a vector field $\bar{L}_{a}^{n}$ on the Poincaré-Lyapunov disc of type $(1,2 l)$. The phase portrait near the equator of this disc (which corresponds to $\infty$, or $s=0$ ) is presented in Figure 1 (a). There are four singularities at $\infty$ : two semi-hyperbolic saddle singularities that we denote by $\mathbf{s}_{ \pm}$in the upper half sphere and two elementary nodes (a repelling and an attracting one) in the lower half-sphere. The semi-hyperbolic singularities $\mathbf{s}_{ \pm}$are connected by a regular orbit $\Gamma_{1}$ at $\infty$ for all $a \in \mathbb{R}^{n-1}$. If for $a^{0}$ arbitrary but fixed there is no connection in the finite plane between $\mathbf{s}_{+}$and $\mathbf{s}_{-}$, then there will be no large amplitude limit cycles bifurcating for $a \rightarrow a^{0}$, see Figure 1 (b). If there does exist a connection $\Gamma_{2}$ in the finite plane between $\mathbf{s}_{+}$and $\mathbf{s}_{-}$, and thus giving rise to an unbounded 2-saddle cycle $\Gamma$, then large amplitude limit cycles can bifurcate from $\Gamma$ for $a \rightarrow a^{0}$, see Figure 1 (c). In the following we assume that such $\Gamma$ exists for $a=a^{0}$, see Figure 3 .

2. Let $\Sigma_{i}$ be a transversal section with respect to $\Gamma_{i}, i=1,2$. As is convenient in the study of limit cycles near a 2 -saddle cycle we define the so-called difference map $\Delta$ as the difference between the transitions $\Delta_{1}$ and $\Delta_{2}$ from $\Sigma_{1}$ to $\Sigma_{2}$, defined by the flow of $\bar{L}_{a}^{n}$ in forward and backward time respectively:

$$
\Delta(w, a)=\Delta_{2}(w, a)-\Delta_{1}(w, a), \text { for } w \geq 0,
$$

where $w$ is a local regular parameter on $\Sigma_{1}$, such that $w=0$ corresponds to $\Gamma_{1} \cap \Sigma_{1}$. In this way isolated zeroes $w$ of $\Delta(\cdot, a)$ for $w \downarrow 0, w \neq 0$ and 


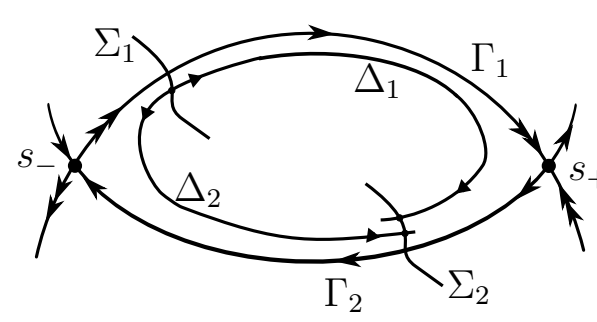

(a)

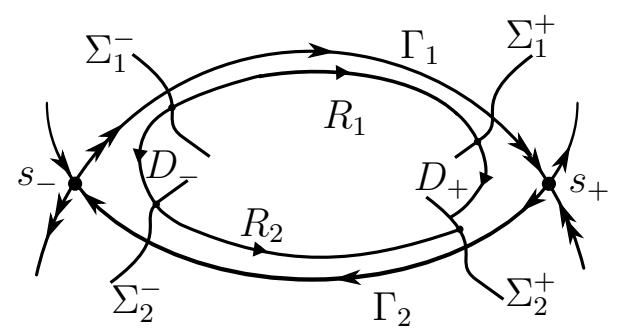

(b)

Figure 3. Difference map $\Delta$ near two-saddle cycle $\Gamma$. (a) $\Delta=$ $\Delta_{2}-\Delta_{1} ;$ (b) $\Delta_{1}=D_{+} \circ R_{1}$ and $\Delta_{1}=R_{2} \circ D_{-}$.

$a \rightarrow a^{0}$ correspond to large amplitude limit cycles of $X_{a}$ near $\Gamma$. Taking appropriate sections $\Sigma_{i}^{ \pm}$with respect to $\Gamma_{i}$ near $\mathbf{s}_{ \pm}$the transition maps $\Delta_{i}$ : $\Sigma_{1}^{-} \rightarrow \Sigma_{2}^{+}, i=1,2$ are written as the composition of a regular transition $R_{i}$ along $\Gamma_{i}$ and a Dulac transition $D_{ \pm}$near the saddle point $\mathbf{s}_{ \pm}$, see Figure 3 (b).

3. To facilitate the calculations near $\Gamma_{1}$ one can use local coordinates $(u, s)$ in which $\bar{L}_{a}^{n}$ reads as

$$
\hat{L}_{a}^{n} \leftrightarrow\left\{\begin{array}{l}
\dot{u}=1-u^{2 l}-G(u, s, a)+(2 l)^{-1} u^{2} s^{4 l-2} \\
\dot{s}=(2 l)^{-1} s^{4 l-1} u
\end{array}\right.
$$

where

$$
G(u, s, a)=\sum_{i=1}^{2 l-1} a_{i} u^{i} s^{2 l-i} .
$$

Clearly $G(u, s, a)=G(-u,-s, a)$; hence $\hat{L}_{a}^{n}$ is invariant with respect to the symmetry $(u, s, t) \mapsto(-u,-s,-t)$, see Figure $4(a)$.

4. By this symmetry we can use the same normalizing coordinates $(z, w)$ near the semi-hyperbolic saddle points $\mathbf{s}_{ \pm}$, transforming $\hat{L}_{a}^{n}$ into the equivalent differential system

$$
X_{a}^{\text {norm }} \leftrightarrow\left\{\begin{array}{l}
\dot{z}=-z, \\
\dot{w}=w^{4 l-1}\left(1+\alpha w^{4 l-2}\right)^{-1},
\end{array}\right.
$$

where $\alpha=\alpha(a)$ depends polynomially on $a$. The Dulac map from $\{z=1\}$ to $\{w=1\}$ near the saddle point $(0,0)$ of $X_{a}^{\text {norm }}$ can be explicitly written down as

$$
D(w)=w^{\alpha} \exp \left(\frac{1-w^{4 l+2}}{4 l-2}\right), \text { for } w \geq 0 .
$$

By the invariance of $X_{a}^{\text {norm }}$ with respect to the symmetry $(z, w, t) \mapsto(z,-w,-t)$ the Dulac map from $\{z=1\}$ to $\{w=-1\}$ near the saddle point $(0,0)$ is given 


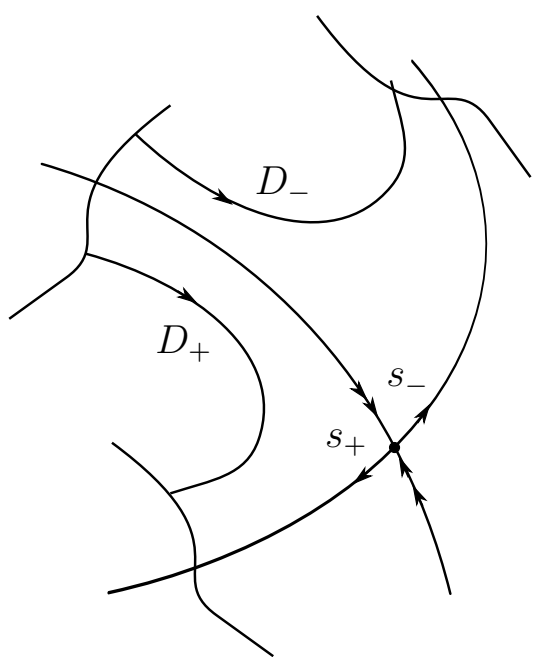

(a)

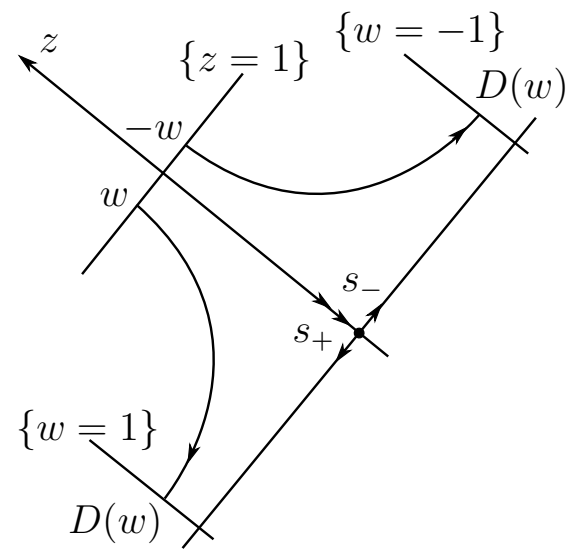

(b)

Figure 4. Dulac transitions $D_{ \pm}$near the saddle points $\mathbf{s}_{ \pm}$using the symmetry (a) $(u, s, t) \mapsto(-u,-s,-t)$ resp. (b) $(z, w, t) \mapsto$ $(z,-w,-t)$, that is present in the coordinates $(u, s, t)$ near $\infty$ resp. the normalizing coordinates $(z, w, t)$.

by $D(-w)$, for $w \leq 0$, see Figures 3 and 4 . By taking the corresponding sections $\Sigma_{i}^{ \pm}$in the $(u, s)$ plane and using these normalizing coordinates as regular parameter one can write

$$
\Delta_{2}(w, a)=R_{2} \circ D(w) \text { and } \Delta_{1}(w, a)=D \circ R_{1}(w) .
$$

For readability, in the notation of $D$ and $R_{i}, i=1,2$ we left out the dependence on the parameter $a$. Clearly $\Delta$ is $C^{\infty}$ at $w=0$ and its jet of infinite order at $w=0$ is given by $j_{\infty}(\Delta)_{0}(w) \equiv R_{2}(0)$, the so-called breaking coefficient. If $R_{2}(0) \neq 0$, then there is no connection between $\boldsymbol{s}_{+}$and $\mathbf{s}_{-}$in the finite plane for the considered parameter $a \in \mathbb{R}^{n-1}$, and hence there are no large amplitude limit cycles for parameter values sufficiently close to $a$.

5. Since there is no explicit expression known for the connection $\Gamma_{2}$ in the finite plane, there is almost no chance to perform an asymptotic analysis of $R_{2}$. Therefore, by taking the derivative and an algebraic manipulation, we replace the study of zeroes of $\Delta(\cdot, a)$ to zeroes of $\Delta^{1}(\cdot, a)$ in such a way that zeroes of $\Delta^{1}(\cdot, a)$ correspond to solutions of $\frac{\partial \Delta}{\partial w}(\cdot, a)=0$. By Rolle's Theorem the number of zeroes $w \downarrow 0$ of $\Delta(\cdot, a)$ is bounded by 1 plus the number of zeroes $w \downarrow 0$ of $\Delta^{1}(\cdot, a)$, where the zeroes are counted with multiplicity. The 
principal part of the so-called reduced difference map $\Delta^{1}$ can be written as:

$$
\Delta^{1}(w, a)=\frac{1}{-4 l+2}\left(\frac{1-\bar{R}_{1}(w, a)}{w}\right)^{-4 l+2}+O\left(w^{4 l-3}\right), w \downarrow 0,
$$

where $R_{1}(w, a)=w \bar{R}_{1}(w, a)$.

6. After some calculations one obtains the asymptotic expansion for $\bar{R}_{1}$ :

$$
\bar{R}_{1}(w, a)=1+a_{2 l-1} w\left(C_{0}+o(1)\right), w \downarrow 0,
$$

for some $C_{0}>0$. Furthermore, if $a_{2 l-1-2 i}^{0}=0, \forall 0 \leq i \leq k-1$, then there exists $C_{k}>0$ such that this asymptotic expansion reads as

$$
\bar{R}_{1}(w, a)=1+a_{2 l-1-2 k} w^{2 k+1}\left(C_{k}+o(1)\right), w \downarrow 0 .
$$

7. Division-derivation algorithm: Assume that $a_{2 l-1-2 i}^{0}=0, \forall 0 \leq i \leq k-1$ for $0 \leq k \leq l-1$. Then combining (4.2), (4.3) and (4.4) we obtain the map $\Delta^{k+1}$ from $\Delta^{1}$ after $k$ derivations and divisions by non-zero functions for $w>0$ with

$$
\Delta^{k+1}\left(w, a^{0}\right)=-a_{2 l-1-2 k}^{0} C_{k}+o(1), w \downarrow 0 .
$$

(a) If $L_{a^{0}}^{n}$ has no center at $(0,0)$, then there exists $0 \leq k \leq l-1$ such that $a_{2 l-1-2 i}^{0}=0, \forall 0 \leq i \leq k-1$ and $a_{2 l-1-2 k}^{0} \neq 0$. Then by continuity $\Delta^{k+1}$ has no zeroes bifurcating from $w=0$ for $a$ sufficiently close to $a^{0}$. By Rolle's Theorem it then follows that there are at most $k+1$ large amplitude limit cycles for $a \rightarrow a^{0}$.

(b) If all $a_{2 l-1-2 k}^{0}=0,1 \leq k \leq l$, then $j_{\infty}\left(\Delta^{1}\left(\cdot, a^{0}\right)\right)_{0}(w) \equiv 0$. However $\Delta^{1}$ is not analytic at $w=0$, and therefore this fact does not imply automatically that $\Delta^{1}\left(\cdot, a^{0}\right) \equiv 0$. However if all $a_{2 l-1-2 k}^{0}=0,1 \leq k \leq l$ then $L_{a^{0}}^{n}$ satisfies a symmetry property implying that $L_{a^{0}}^{n}$ has a center at $(0,0)$, and hence $\Delta^{1}\left(\cdot, a^{0}\right) \equiv 0$. Applying Taylor's Theorem $\Delta^{1}$ can locally be written as:

$$
\Delta^{1}(w, a)=\sum_{k=1}^{l} a_{2 l-2 k-1} \Phi_{k}(w, a),
$$

for $C^{\infty}$ functions $\Phi_{k}, 1 \leq k \leq l$ with

$$
\Phi_{k}(w, a)=C_{k} w^{2 k}(1+o(1)), w \downarrow 0 .
$$

By the following rescaling of the parameter we can reduce this degenerate case to the regular case:

$$
\begin{gathered}
(b, \rho)=\left(b_{1}, \ldots, b_{2 l-1}, \rho\right) \mapsto a, \text { where } \forall 1 \leq i \leq l: \\
a_{2 i}=b_{2 i}, \quad a_{2 i-1}=\rho b_{2 i-1} \text { and } \sum_{i=1}^{l} b_{2 i-1}^{2}=1 .
\end{gathered}
$$


In terms of the rescaled parameter $(b, \rho)=\left(b_{1}, \ldots, b_{2 l-1}, \rho\right)$ we then have

$$
\Delta^{1}(w, a)=\rho \sum_{k=1}^{l} b_{2 l-2 k-1} \Phi_{k}(w, a)=\rho \bar{\Delta}^{1}(w, b, \rho) .
$$

For each $b \in \mathbb{R}^{n-1}$ fixed with $\sum_{i=1}^{l} b_{2 i-1}^{2}=1$, we can apply the divisionderivation algorithm to $\bar{\Delta}^{1}$ and find that there are at most $l+1$ large amplitude limit cycles that bifurcate from $\Gamma$ for $a \rightarrow a^{0}$.

\subsection{Putting a boundary on the space of Liénard equations}

Following the idea of Roussarie in [27] we put a boundary on the Liénard family $\left(L_{a}^{n}\right)_{a \in \mathbb{R}^{n-1}}$ to end up with a compact family. More precisely we imbed $\left(L_{a}^{n}\right)_{a \in \mathbb{R}^{n-1}}$ in the family $\left(S_{a, \varepsilon}^{n}\right)$ that is defined as

$$
S_{a, \varepsilon}^{n} \leftrightarrow \dot{x}=y-F_{a}^{n}(x), \dot{y}=-\varepsilon x \text { for } \varepsilon>0 .
$$

Clearly $L_{a}^{n} \equiv S_{a, 1}^{n}$. Furthermore $S_{a, 0}^{n}$ is a singular family of planar vector fields, having the graph of $y=F_{a}^{n}(x)$ full of non-isolated singularities; the vector fields of the family $S_{a, \varepsilon}^{n}$ for $\varepsilon>0$ small are called slow-fast Liénard equations.

For later use let us introduce the natural projections on $\mathbb{R}^{n}=\mathbb{R}^{n-1} \times \mathbb{R}$ by $\pi_{1}: \mathbb{R}^{n-1} \times \mathbb{R} \rightarrow \mathbb{R}^{n-1}:(a, \varepsilon) \mapsto a$ and $\pi_{2}: \mathbb{R}^{n-1} \times \mathbb{R} \rightarrow \mathbb{R}:(a, \varepsilon) \mapsto \varepsilon$.

The family $\left(S_{a, \varepsilon}^{n}\right)_{a \in \mathbb{R}^{n-1}, \varepsilon>0}$ is quasi-homogeneous; as a consequence for all $(a, \varepsilon)$ and $(\bar{x}, \bar{y})$ fixed, there exist 1-parameter groups of diffeomorphisms

$$
\left\{T_{\tau}(\bar{x}, \bar{y}): \tau \in \mathbb{R}\right\} \text { and }\left\{U_{\tau}(a, \varepsilon): \tau \in \mathbb{R}\right\}
$$

such that $S_{U_{\tau}(a, \varepsilon)}^{n}$ is conjugate to $\tau^{n-1} S_{a, \varepsilon}^{n}$ via $T_{\tau}$, where

$$
\begin{aligned}
& T_{\tau}(\bar{x}, \bar{y})=\left(\tau \bar{x}, \tau^{n} \bar{y}\right) \text { and } \\
& U_{\tau}(a, \varepsilon)=\left(\tau^{n-1} a_{1}, \tau^{n-2} a_{2}, \ldots, \tau a_{n-1}, \tau^{2 n-2} \varepsilon\right) \text { for } a=\left(a_{1}, \ldots, a_{n-1}\right) .
\end{aligned}
$$

The phase portraits of $S_{U_{\tau}(b, \delta)}^{n}$ and $S_{b, \delta}^{n}$ thus are diffeomorphic via $T_{\tau}$, and so $S_{U_{\tau}(b, \delta)}^{n}$ and $S_{b, \delta}^{n}$ have the same number of limit cycles in the plane.

Let $\|\cdot\|$ be the Euclidean norm on $\mathbb{R}^{n-1}$, and define the unit disc and its boundary respectively by

$$
\Delta \equiv\left\{a \in \mathbb{R}^{n-1}:\|a\| \leq 1\right\} \text { and } \partial \Delta \equiv\left\{a \in \mathbb{R}^{n-1}:\|a\|=1\right\} .
$$

Then for any $\varepsilon>0$ the family $\left(S_{a, \varepsilon}^{n}\right)_{a \in \Delta}$ is equivalent to the bounded Liénard family $\left(L_{b}^{n}\right)_{b \in K_{\varepsilon}}$ via $\tau(\varepsilon)$, where

$$
K_{\varepsilon} \equiv\left\{\pi_{1}\left(U_{\tau(\varepsilon)}(a, \varepsilon)\right):\|a\| \leq 1\right\} \text { and } \tau(\varepsilon) \equiv \sqrt[2 n+2]{1 / \varepsilon} \text { for } \varepsilon>0 .
$$

In particular one has $\pi_{2}\left(U_{\tau(\varepsilon)}(a, \varepsilon)\right)=1$ and for $\varepsilon \downarrow 0$ the diameter of $K_{\varepsilon}$ tends to $\infty$, see Figure 5. Indeed notice that the 1-parameter groups of smooth diffeomorphisms $\left\{U_{\tau}(a, \varepsilon), \tau>0\right\}$ for fixed values $(a, \varepsilon)$ can be defined by the differential 


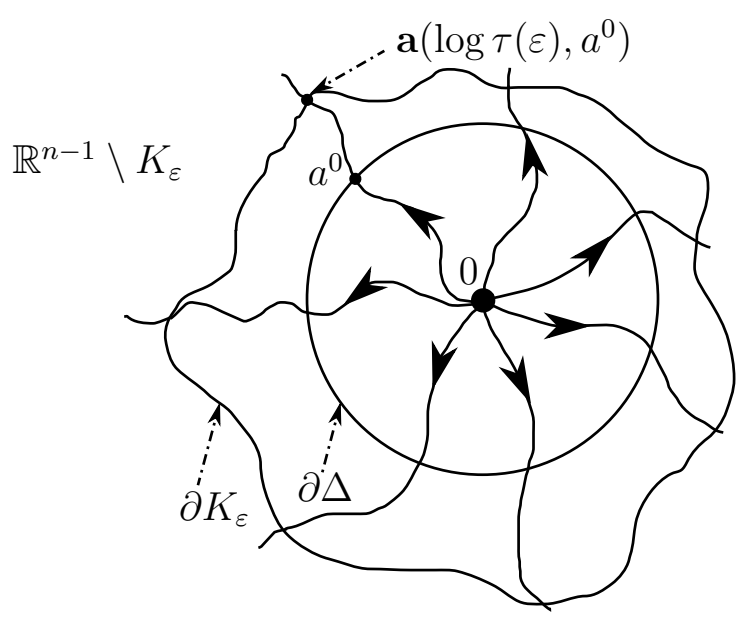

Figure 5. Bounded versus unbounded Liénard equations $L_{a}^{n}$. Here $\mathbf{a}\left(\cdot, a^{0}\right)$ denotes the flow defined by (4.8) with initial condition $a=a^{0}$; the time $\log (\tau(\varepsilon))$ is necessary to pass from $\varepsilon$ to 1 . Since (4.8) describes a linear expansion the compact set $K_{\varepsilon} \uparrow \mathbb{R}^{n-1}$ for $\varepsilon \downarrow 0$, see (4.7).

equations

$$
\frac{\mathrm{d}}{\mathrm{d} \log \tau} a_{i}=(n-i), \text { for all } 1 \leq i \leq n-1 \text { and } \frac{\mathrm{d}}{\mathrm{d} \log \tau} \varepsilon=(2 n-2)
$$

in parameter space $\mathbb{R}^{n}$. Furthermore the orbits of (4.6) are transverse to the hyperspace $\{\varepsilon=1\}$; therefore and since the equations for the parameter variable $a \in \mathbb{R}^{n-1}$ are independent from the one for $\varepsilon>0$, we can study the evolution of the disc $\Delta$ and its boundary $\partial \Delta$ in the projected space $\mathbb{R}^{n-1}$ by $\pi_{1}$ after time $\log \tau(\varepsilon)$, which is strictly increasing to $+\infty$ when $\varepsilon \downarrow 0$ (see Figure 5).

Therefore for any fixed $\varepsilon_{0}>0$ the family $\left(S_{a, \varepsilon_{0}}^{n}\right)_{a \in \Delta}$ is equivalent to a 'bounded Liénard family' $\left(L_{b}^{n}\right)_{b \in K_{\varepsilon_{0}}}$ and the family $\left(S_{a, \varepsilon}^{n}\right)_{a \in \partial \Delta, 0<\varepsilon<\varepsilon_{0}}$ is equivalent to its complement in the space of Liénard equations $\left(L_{b}^{n}\right)_{b \in \mathbb{R}^{n-1}}$, i.e. $\left(L_{b}^{n}\right)_{b \in \mathbb{R}^{n-1} \backslash K_{\varepsilon_{0}}}$, which is unbounded (see Figures 5 and 6).

It follows from the results in sections 4.3 and 4.4 that for any fixed $\varepsilon_{0}>0$ there exists an integer $N\left(n, \varepsilon_{0}\right)$ such that the number of limit cycles of the bounded Liénard family $\left(L_{b}^{n}\right)_{b \in K_{\varepsilon_{0}}}$ is uniformly bounded by $N\left(n, \varepsilon_{0}\right)$ in the plane. Therefore to complete Smale's 13th Problem it suffices to solve the finiteness problem for the compact family of polynomial vector fields $\left(S_{a, \varepsilon}^{n}\right)_{a \in \partial \Delta, 0 \leq \varepsilon \leq \varepsilon_{0}}$, where $\varepsilon_{0}>0$ can be taken as small as necessary. Notice that for $\varepsilon_{0} \downarrow 0$ limit cycles of $S_{a, \varepsilon}^{n}$ shrink to the origin. As a consequence, using the compactness of $\partial \Delta$ and the localization method of Roussarie, Smale's 13th Problem is reduced to a singular $\varepsilon$-perturbation 


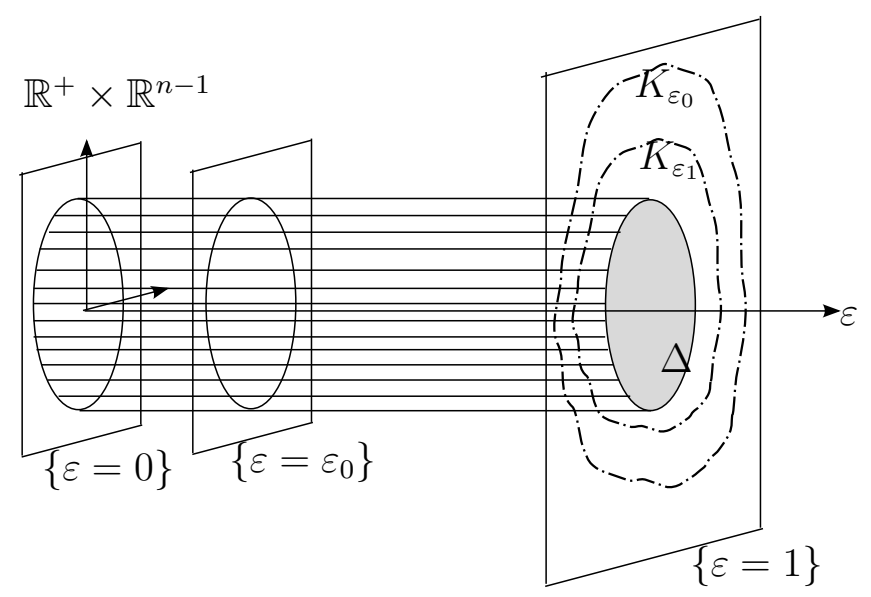

Figure 6. Compactification of classical Liénard equations $\left\{L_{b}^{n}\right.$ : $\left.b \in \mathbb{R}^{n-1}\right\}$. The constants $\varepsilon_{i}>0, i=0,1$ are assumed to be ordered as $\varepsilon_{0}<\varepsilon_{1}$. The bounded Liénard family $\left\{L_{b}^{n}: b \in K_{\varepsilon_{0}}\right\}$ is equivalent to $\left\{S_{a, \varepsilon_{0}}^{n}: a \in \Delta\right\}$ and the unbounded Liénard family $\left\{L_{b}^{n}: b \in \mathbb{R}^{n-1} \backslash K_{\varepsilon_{0}}\right\}$ is equivalent to $\left\{S_{a, \varepsilon}^{n}: a \in \partial \Delta, 0 \leq \varepsilon<\varepsilon_{0}\right\}$.

problem: the cyclicity problem of the singular point $(0,0)$ of the singular vector field $S_{a^{0}, 0}^{n}$ inside the family $\left(S_{a^{0}, \varepsilon}^{n}\right)_{0 \leq \varepsilon \leq \varepsilon_{0}}$ for all $a^{0} \in \partial \Delta$, where $\varepsilon_{0}$ is sufficiently small (but independent of $a^{0}$ ).

\subsection{Conclusions and some generalizations}

In sections $4.1,4.2, \ldots, 4.5$ we have sketched the proof of Theorem 2.1, i.e., how Hilbert's 16th Problem is solved for bounded classical Liénard equations, and how Smale's 13th Problem is reduced to some cyclicity problems for slow-fast systems. Besides this proof includes an independent proof of the Dulac Theorem for classical Liénard equations.

In [19] the Hilbert number for the bounded family of classical Liénard equations of even degree $n$ in case the origin is a focus, say $\mathcal{H}_{B L}^{*}(1, n)$, is estimated in terms of different parameters using the Growth-and-Zeroes Theorem provided by Ilyashenko and Yakovenko and the result on large amplitude limit cycles from [4].

For a complete study of large amplitude limit cycles for the family of generalized Liénard equations, i.e., (1.2) when $g(x) \neq x$, the characterization of an unbounded center is needed; this is detailed in [5] where complete results only are obtained for certain subfamilies of generalized Liénard equations.

\section{Acknowledgment}

The author would like to thank the organizers A. Gasull, J. Hartmann, J. Llibre and S. Walcher for this invitation at the Oberwolfach Mini-Workshop where they 
brought together a lot of interesting talks both on qualitative study of polynomial vector fields as well as on algebraic and analytic geometry leaving time for stimulating discussions on interesting and important open problems.

\section{References}

[1] M. Bobienski and H. Zoladek, A Counterexample to a multidimensional version of the Weakened Hilbert's 16th Problem. Moskow Mathematical Journal 7 (2007), 1-20.

[2] C.J. Christopher, Estimating Limit Cycles Bifurcations from Centers. in: Trends in Mathematics: Differential Equations with Symbolic Computation, Birkhäuser Verlag Basel/Switzerland, pp.23-35, 2006.

[3] M. Caubergh and F. Dumortier, Hopf-Taken bifurcations and centres. Journal of Differential Equations 202 (2004), 1-31.

[4] M. Caubergh and F. Dumortier, Hilbert's 16th problem for classical Liénard equations of even degree. Journal of Differential Equations 244 (2008), 1359-1395.

[5] M. Caubergh, F. Dumortier and S. Luca, Cyclicity of unbounded semi-hyperbolic 2saddle cycles in polynomial Liénard systems. Discrete and Continuous Dynamical Systems 27 (2010), 963-980.

[6] M. Caubergh and J.P. Françoise, Generalized Liénard equations, cyclicity and HopfTakens bifurcations. Qualitative Theory of Dynamical Systems 5(2004), 195-222.

[7] C.J. Christopher and N.G. Lloyd, Polynomial Systems: A Lower Bound for the Hilbert Numbers. Proceedings: Mathematical and Physical Sciences 450 (1995), 219-224.

[8] W. A. Coppel, Some Quadratic Systems with at Most One Limit Cycle. Dynamics Reported 2 (1988), Wiley, New York, 61-68.

[9] L. Chen and M. Wang, Relative position and number of limit cycles of a quadratic differential system. (Chinese), Acta Mathematica Sinica 22(1979), 751-758.

[10] P. De Maesschalck and F. Dumortier, Classical Liénard equations of degree $n \geq 6$ can have $[(n-1) / 2]+2$ limit cycles. Journal of Differential Equations 250, 2162-2176.

[11] F. Dumortier and C. Herssens, Polynomial Liénard Equations near Infinity. Journal of Differential Equations 153 (1999), 1-29.

[12] F. Dumortier and C. Li, On the uniqueness of limit cycles surrounding one or more singularities for Liénard equations. Nonlinearity 9 (1996), 1489-1500.

[13] F. Dumortier and C. Li, Quadratic Liénard Equations with Quadratic Damping. Journal of Differential Equations 139 (1997), 41-59.

[14] F. Dumortier and C. Li, Perturbation from an elliptic Hamiltonian of degree four: (IV) Figure eight-loop. Journal of Differential Equations 188 (2003), 512-554.

[15] F. Dumortier, D. Panazzolo and R. Roussarie, More limit cycles than expected in Liénard equations. Proceedings of the American Mathematical Society 135 (2007), 1895-1904

[16] F. Dumortier and R. Roussarie, Birth of canard cycles. Discrete and Continuous Dynamical Systems S 2 (2009) 723-781.

[17] F. Dumortier and C. Rousseau, Cubic Liénard equations with linear damping. Nonlinearity 3 (1990), 1015-1039. 
[18] Yu. S. Ilyashenko, Centennial history of Hilbert's 16th Problem. Bulletin of the American Mathematical Society (N.S.) 39 (2002), 301-354.

[19] G. Kolutsky, An upper estimate for the number of limit cycles of even-degree Liénard equations in the focus case. Journal of Dynamical and Control Systems 17 (2011), 231-241.

[20] J. Li, H.S.Y. Chan and K.W. Chung, Some Lower Bounds for H(n) in Hilbert's 16th Problem. Qualitative Theory of Differential Equations 3 (2003), 345-360.

[21] N.G. Lloyd and S. Lynch, Small-amplitude limit cycles of certain Liénard systems. Proceedings of the Royal Society London A, 418 (1988), 199-208.

[22] C. Li and J. Llibre, Uniqueness of Limit Cycle for Liénard Equations of Degree Four. Preprint, 2011.

[23] C. Li, C. Liu and J. Yang, A cubic system with thirteen limit cycles. Journal of Differential Equations 246 (2009), 3609-3619.

[24] A. Lins, W. de Melo and C.C. Pugh, On Liénard's equation. Lecture Notes in Mathematics 597 (1977), 335-357, Springer, Berlin.

[25] J. Li, M. Zhang and S. Li, Bifurcations of limit cycles in a $Z_{2}$-equivariant planar polynimial vector field of degree 7 . International Journal of Bifurcation and Chaos $\mathbf{1 6}$ (2006), 925-943.

[26] R. Roussarie, Bifurcations of Planar Vector Fields and Hilbert's Sixteenth Problem. Progress in Mathematics 164, Birkhauser-Verlag, Basel, 1998.

[27] R. Roussarie, Putting a boundary to the space of Liénard Equations. Discrete and Continuous Dynamical Systems 17 (2007), 441-448.

[28] S. Smale, Mathematical Problems for the Next Century. Mathematical Intelligencer 20 (1998), 7-15.

[29] Shi Songling, A concrete example of the existence of four limit cycles for plane quadratic systems. Scientia Sinica 23 (1980), 154-158.

[30] Y. Wu, Y. Gao and M. Han, On the number and distributions of limit cycles in a quintic planar vector field. International Journal of Bifurcation and Chaos in Applied Sciences and Engineering, 18 (2008), 1939-1955.

[31] S. Wang and P. Yu, Bifurcation of limit cycles in a quintic Hamiltonian system under a sixth-order perturbation. Chaos, Solitons and Fractals 26(5) (2005), 1317-1335.

[32] J. Yang, M. Han and V.G. Romanovski, Limit cycle bifurcations of some Liénard systems. Journal of Mathematical Analysis and Applications, 336 (2010), 242-255.

[33] Zhang Zhi-fen, Ding Tong-ren, Huang Wen-zoa, Dong Zhen-xi. Qualitative Theory of Differential Equations. American Mathematical Society, Providence, 1992.

Magdalena Caubergh

Departament de Matemàtiques

Facultat de Ciències(Edifici C)

Universitat Autònoma de Barcelona

Campus Bellaterra

09381 Cerdanyola del Vallès (Barcelona)

Spain

e-mail: leen@mat.uab.cat 